\title{
HISTORIA DE LA EDUCACIÓN BILINGÜE EN EL ESTADO DE NUEVO MÉXICO
}

Fernando Martín Pescador

atravesdelespejo@hotmail.com

\section{RESUMEN}

Nuevo México ha sido siempre un estado plurilingüe y multicultural. Por lo tanto, la educación en el estado siempre ha necesitado un componente de bilingüismo. Los gobiernos de España, México y Estados Unidos tuvieron que adaptar sus sistemas educativos para poder satisfacer las necesidades de la población local. El esfuerzo del pueblo nuevomexicano ha sido constante a lo largo de toda la historia para mantener vivas todas sus lenguas. Ese esfuerzo aparece reflejado en su Constitución y en lo pioneras que han sido sus leyes de educación dentro de los Estados Unidos e incluso en el contexto internacional. Este artículo repasa los momentos, los movimientos y las leyes más importantes que han ido marcando la historia de la educación bilingüe en Nuevo México.

Palabras clave: Educación Bilingüe, Nuevo México, suroeste EEUU, bilingüismo, historia de la educación.

\section{AbStract}

New Mexico has always been a multilingual and multicultural state. Hence the Education policies in this state have always required a bilingual focus. The governments of Spain, Mexico and the United States had to adapt their educational systems in order to meet the needs of New Mexico. New Mexicans' effort to keep all their languages alive has been a constant struggle throughout history. It was reflected on their Constitution and on their trailblazing education laws within the US and even in an international context. 
This article goes over the milestones, social movements and State and Federal laws that have made an important impression on Bilingual Education in New Mexico.

KeY words: Bilingual Education, New Mexico, US Southwest, Bilingualism, History of Education.

Uno de los factores determinantes para preservar el bilingüismo en Nuevo México ha sido, es y será la política educativa del estado en enseñanza bilingüe. El español o cualquiera de las lenguas nativoamericanas no podrán sobrevivir de la forma que lo han hecho hasta ahora por la sencilla razón de que el inglés, la lengua de prestigio en el estado, llega a cada pulgada del territorio.

Si estas lenguas históricas no son enseñadas y utilizadas formalmente en las escuelas, acabarán desapareciendo del territorio nuevomexicano. Sería una pérdida cultural devastadora, especialmente en el caso de las lenguas nativoamericanas puesto que no se hablan en ningún otro lugar del mundo.

De la misma forma que Nuevo México ya no está aislado del mundo, serán de vital importancia las políticas educativas que se potencien desde Washington. Tradicionalmente, los estados han sido muy celosos a la hora de conservar su independencia en política educativa pero la ley de No Child Left Behind $(2002)^{1}$ ha dado unas cuotas de protagonismo al gobierno federal sin precedentes ${ }^{2}$. Nunca hasta ahora, tampoco, había habido unos benefactores privados de la enseñanza con un volumen de subvenciones tal que su capacidad de influencia a la hora de dictar leyes de educación estatales sea tan decisiva ${ }^{3}$.

La educación bilingüe en el estado ha fracasado en muchos sentidos en los últimos cincuenta años, empezando por no convencer a la sociedad de todas sus virtudes. Sin embargo, hay motivos para el optimismo. Las nuevas tendencias educativas de programas de inmersión dual en las escuelas promueven una enseñanza pública en la que los estudiantes aprenden en dos lenguas y ambas son reconocidas como lenguas de prestigio. Los programas de educación de inmersión dual conciencian a la comunidad educativa y a los muchachos de que en el siglo XXI saber dos lenguas es fundamental para mantener viva la cultura de nuestros antepasados y para triunfar en un mercado de trabajo cada vez más competitivo.

A lo largo de estas páginas, repasaremos algunos de los momentos más importantes en la historia de la educación en Nuevo México. Una educación que, como veremos, no ha dejado nunca de tener un componente plurilingüe y multicultural.

Antes de la llegada de los españoles, en Nuevo México no existía una lengua franca con la que los diversos pueblos pudieran comunicarse. El comercio entre los pueblos era frecuente con lo que muchos nativoamericanos se veían con la necesidad de saber expresarse en varias lenguas. En esos momentos, había unos 90.000 habitantes en la

\footnotetext{
${ }^{1}$ La ley No Child Left Behind (Que ningún niño se quede atrás) fue la ley de Educación del gobierno de George W. Bush y sigue vigente en 2014.

2 Diane Ravitch (2010): The Death and Life of the Great American School System. How Testing and Choice Are Undermining Education, Nueva York: Basic Books, p. 94.

3 Ibídem, pp. 195-222.
} 
región y se hablaban el tehua, el tigua, el towa, el kerés y el zuñi además de dos lenguas ya extinguidas: el piro y el tampiro. La educación en los pueblos se llevaba a cabo de forma comunitaria, a través de la iniciación de los muchachos, de las historias orales y de los cantos tradicionales.

En 1523, Felipe II proclama que todos los religiosos que marchen a América deben aprender las lenguas de los nativos de México y de los territorios del norte y les ordena que enseñen las sagradas escrituras en la lengua que más fácil resulte a los nativos ${ }^{4}$. Los franciscanos escribieron gramáticas y diccionarios de varias lenguas, tradujeron crónicas nativoamericanas al español y tradujeron oraciones españolas al náhuatl ${ }^{5}$.

Con la llegada de los españoles a Nuevo México, llegaron los primeros profesores a Nuevo México. Fray Juan de Padilla y Fray Luis de Escalona llegaron con la expedición de Coronado en 1540 y decidieron quedarse cuando éste comenzó su vuelta a Nueva España. El primero en Quivera y el segundo en Cicuye. Los dos encontraron primero buena disposición pero fueron luego asesinados por sus estudiantes. Los padres franciscanos tuvieron que aprender la lengua del pueblo en el que se establecieron.

En 1626 y 1630, Fray Alonso de Benavides escribió una serie de informes para el rey de España en los que hablaba de las misiones y, dentro de estos informes, contaba al rey sobre las escuelas. Estaba claro que la conversión al cristianismo era el objetivo principal de los franciscanos pero, en las escuelas, también se enseñaba a leer, a escribir, música y varios oficios. Los indios Pueblo podían aprender a coser, a hacer zapatos, a trabajar el metal y la madera así como a pintar. En 1680 había 46 pueblos con una escuela en la misión o un centro de aprendizaje 6 .

La coexistencia de tantas lenguas provocaba desasosiego en algunos gobernantes (como el que expresaba el virrey Don Luis de Velasco escribiendo al gobernador Pedro de Peralta en 1609) y, desde el poder, también se intentaba enseñar español a todos los habitantes del nuevo territorio:

\footnotetext{
${ }^{4}$ Hará lo mismo el 21 de octubre de 1595 cuando dé permiso para la conquista de Nuevo México en el nombramiento de Juan de Oñate como gobernador y capitán general de Nuevo México:

«Les explicaréis nuestra Sagrada Fe Católica a través de intérpretes, si se pueden conseguir, para que podamos comunicarnos con ellos en sus diversas lenguas.»

Véase en Thomas E. Chávez (2002) [1992]: An Illustrated History of New Mexico. Albuquerque, University of New Mexico Press, p. 42:

Texto original:

"You will explain our holy Catholic faith to them through interpreters, if they can be obtained, so that we may have communication with them in the various languages and seek their conversations.»

${ }_{5}$ Bills and Vigil explican la importancia del náhuatl en Bills, Garland D. \& Vigil, Neddy A. (2008): The Spanish Language of New Mexico and Southern Colorado: A Linguistic Atlas. Albuquerque, University of New Mexico Press, p. 93:

Los aztecas se habían convertido en la más poderosa de las civilizaciones nativomexicanas y habían impuesto su poder por gran parte del centro de México. Su lengua, el náhuatl, era el idioma nativoamericano dominante de la región en ese momento, reflejando el poder político y económico de los aztecas. Tendría sentido, por lo tanto, que su influencia sobre la lengua española fuera importante.

Texto original:

The Aztecs had become the most powerful of a number of Mexican Indian civilizations and had imposed their will throughout much of central Mexico. Their language, Nahuatl, was the dominant Native American language of the region at that time, representing the political and economic power of the Aztecs. We could anticipate, therefore, that its influence on Spanish would be important.

${ }^{6}$ John B. Mondragón \& Ernest S. Stapleton (2005): Public Education in New México. Albuquerque:, University of New Mexico, pp. 3-5.
} 
Según parece, Nuevo México alberga varios idiomas muy difíciles y bárbaros que causan más de un inconveniente para conseguir una buena administración y poder ayudar a los religiosos y a los nativos. El mencionado gobernador debe actuar con mucho cuidado consultando a los sacerdotes de tal manera que enseñen a todos los indios y especialmente a los niños y a los ignorantes para que puedan aprender español y en caso de que no puedan aprender el mismo, un mismo esfuerzo debe al menos ser hecho para que aquellos que no tengan habilidad natural para aprender español aprendan un idioma que sea hablado por el mayor número posible de personas en el lugar para que puedan así ser gobernados más eficientemente. $^{7}$

Sin embargo, las diversas lenguas mantenían su protagonismo. En el siglo XVII, aparece en la escena nuevomexicana María de Ágreda, una monja española con el don de la ubicuidad. A la vez que escribía en el convento de Ágreda (Soria), se aparecía a los nativos pidiéndoles que se convirtieran a la fe católica en cada una de sus lenguas (como describe Fray Alonso de Benavides, describiendo las apariciones de María de Ágreda a los indios en 1630 :

Preguntamos a los indios por qué tenían tantas ganas de ser bautizados y de que los frailes les enseñaran la doctrina cristiana. Respondieron que una mujer, similar a la que teníamos pintada allí, les decía, a cada uno en su propia lengua, que vinieran sin demora y pidieran a los sacerdotes que les enseñaran y los bautizaran. ${ }^{8}$

En 1721, siguiendo un decreto del rey de España, fueron creadas las primeras escuelas públicas de Nuevo México. Cada pueblo debía cultivar un campo de trigo y pagar con sus frutos al maestro de la escuela. Pocas poblaciones siguieron las órdenes de este decreto.

Pedro Baptista Pino, en su informe a la Corona con el que viajó a las Cortes de Cádiz de 1812 se queja de la situación de la educación en el estado y del pobre apoyo económico que España ofrece a un territorio que ha luchado durante siglos para mantener la frontera del Imperio intacta:

El de las escuelas de primeras letras está reducido á que los que tienen facultades para contribuir al maestro pueden enseñar á sus hijos: en la misma capital no se ha podido dotar un maestro para hacer común enseñanza.

${ }^{7}$ Citado en Thomas E. Chávez (2002) [1992]: An Illustrated History of New Mexico. Albuquerque, University of New Mexico Press, p. 46.

Texto original:

It is understood that that country [New Mexico] is settled by various languages very difficult and barbarous which cause many inconveniences for the good administration and consolation of the ministers as well as of the natives. The said Governor is requested to act with great care consulting with the religious in such a manner so that the main thing shall be to teach all the Indians and especially the children and ignorant persons so they may learn the Spanish language and in the event that they cannot learn the same generally an effort must at least be made that those who have no natural fitness to learn the Spanish language must be taught the language most generally spoken in that country so that they may be better administrated.

${ }^{8}$ Citado en Thomas E. Chávez (2002) [1992]: An Illustrated History of New Mexico. Albuquerque, University of New Mexico Press, p. 54.

Texto original:

We asked the Indians why they were so zealous in asking for Baptism and for friars to go to instruct them in Christian doctrine. They replied that a woman, similar to the one whose likeness we had there painted, used to tell them, each in his own language, to come without delay and summon the priests that they might teach and baptize them.» 
Por supuesto que tampoco hai colegio alguno de estudios: de aquí viene el desconsuelo que manifiestan muchas personas que advierten la buena disposición de los hijos de la provincia para las ciencias, y en mas de 200 años de su conquista no cuenta uno colocado en ninguna carrera literaria, ni aun de sacerdote; cosa tan común en las demás provincias de América. ${ }^{9}$

Las leyes de 1822 y 1823 intentaron establecer las escuelas públicas bajo el mandato mexicano. La ley hablaba sobre todo de la escuela primaria pero en 1823, se intentó crear una escuela secundaria ya que la escuela secundaria de El Paso era la única en todo el territorio. En 1826 se abre la Escuela Superior (College) de Santa Fe. El padre Antonio José Martínez funda una escuela en Taos en el mismo año. Antonio José Martínez establece la primera imprenta de Nuevo México en 1834 y escribe una gramática, Cuaderno de Ortografía ${ }^{10}$, que se convierte en el primer libro de texto para las escuelas publicado en Nuevo México.

A pesar de todos estos primeros pasos, en 1840, aparecen en el censo tan sólo 18 maestros. Las familias pudientes mandaban a sus hijos a Durango o a Saint Louis o incluso a Nueva York ${ }^{11}$. Si así estaba la situación de la educación entre los mexicanos, en los Pueblos, no podía estar mucho mejor. Estas son las impresiones de Josiah Gregg en una de las entradas de su diario entre 1831 y 1839 :

Aunque los Pueblos son famosos por su hospitalidad y su talento, todavía continúan en el más salvaje estado de ignorancia, sin libros ni escuelas, a la vez que ninguno de sus idiomas ha sido reducido a unas reglas gramaticales y a muy pocos de sus niños se les enseña en español. ${ }^{12}$

Cuando el gobernador Martínez vino de México a Santa Fe en 1844, trajo a un maestro con él, Francisco Gonzales, y contrató a otro, un inglés llamado Edward Papy. En 1847, en su primer discurso ante la Legislatura de Nuevo México, el gobernador Vigil dijo:

Hay en la actualidad una sola escuela pública en el territorio, aquella localizada en la ciudad de Santa Fe, y financiada con los presupuestos del condado, los cuales no dan para contratar a más de un maestro. ${ }^{13}$

9 Pedro Baptista Pino (1995) [1812]: Exposición sucinta y sencilla de la provincia del Nuevo Mexico: Hecha por su diputado en Córtes Don Pedro Baptista Pino con arreglo a sus instrucciones, Santa Fe y Albuquerque, El Rancho de las Golondrinas y University of New Mexico Press, pp. 18-19.

10 Antonio José Martínez (1834): Cuaderno de Ortografía. Dedicado a los niños de los señores Martines de Taos, Santa Fe, Imprenta de Ramón Abreu a cargo de Jesús María Baca. Se puede ver una copia virtual del Cuaderno de Ortografía en la página web de la Biblioteca Digital Mundial de la Unesco: http://www.wdl.org/ es/item/78/. Acceso el 26 de abril de 2014.

${ }^{11}$ John B. Mondragón \& Ernest S. Stapleton (2005): Public Education in New México. Albuquerque, University of New Mexico, pp. 8-9.

12 Josiah Gregg (2006) [1845]: Gregg's Commerce of the Prairies or The Journal of a Santa Fe Trader, 1831-1839. Carlile, Massachusets, Applewood Books, p. 61.

Texto original:

Although the Pueblos are famous for hospitality and industry, they still continue in the rudest state of ignorance, having neither books nor schools among them, as none of their languages have been reduced to rules, and very few of their children are ever taught in Spanish.

${ }^{13}$ George P. Hammond \& Thomas C. Donnelly (1941) [1936]: The Story of New Mexico. Albuquerque, University of New Mexico Press, pp. 127-128.

Con toda seguridad, el gobernador Vigil pronunció estas palabras en español. Nosotros sólo tenemos la cita en inglés y la traducción que ofrecemos arriba es del autor: 
En 1848, el Tratado de Guadalupe-Hidalgo otorgaba la nacionalidad estadounidense a los mexicanos que se quedaran en los estados del suroeste que pasaban entonces a manos de Washington ${ }^{14}$. No sólo eso, dicho tratado se convirtió en una carta de derechos para los nuevos habitantes de la Unión que se enarbola todavía hoy a la hora de defender el bilingüismo y la educación bilingüe en Nuevo México. Podemos hablar, sin reparos, de que el espíritu del Tratado de Guadalupe-Hidalgo tiene todavía en el siglo XXI mucha fuerza. Poco ayudó el gobierno central a que los mexicanos defendieran sus tierras de los nuevos especuladores que llegaban del este; muchos otros puntos del tratado no fueron respetados por Washington. Sin embargo, en los momentos más duros de las leyes racistas de inmigración de los Estados Unidos, en los que ni siquiera los hijos de orientales nacidos en el país tenían derecho a la nacionalidad estadounidense, los mexicanos pudieron protegerse gracias al tratado ${ }^{15}$.

En el mismo año en el que se firma el Tratado de Guadalupe-Hidalgo, J. W. Dunn abrió la primera escuela del estado en la que se enseñaba la lengua inglesa. Puso un anuncio en el Republican de Santa Fe, el primer periódico en inglés del Estado y la denominó Classical and High School (Escuela secundaria y de estudios clásicos) ${ }^{16}$.

Nuevo México es ya territorio de los Estados Unidos y, por lo tanto, la iglesia católica del estado deja de depender de los arzobispados mexicanos. El primer obispo que se encarga del estado bajo el nuevo gobierno fue el francés Jean Baptiste Lamy. La decisión no gustó entre el clero nuevomexicano puramente hispanohablante. Los enfrentamientos fueron especialmente duros entre el nuevo obispo y el padre Antonio José Martínez, que acabó siendo excomulgado debido a una de las disputas.

A pesar de las grandes rivalidades entre ellos, ambos se preocuparon muchísimo de la educación en Nuevo México. Jean Baptiste Lamy potenció la apertura de nuevas escuelas católicas y abrió St. Michael's College en Santa Fe (abriendo las puertas a otra congregación francesa, la de los hermanos de La Salle) en $1859^{17}$. Para algunos críticos, sin embargo, apoyó en exceso las escuelas parroquiales católicas y con sus políticas retrasó el establecimiento de una escuela pública estatal ${ }^{18}$.

«There is at present but one public school in the territory, that located in the city of Santa Fe, and supported by the funds of the county, which are insufficient to employ more than one teacher.»

${ }_{14}$ Tratado de Guadalupe-Hidalgo del 2 de febrero de 1848, Artículo VIII (podemos encontrar el tratado de Guadalupe-Hidalgo en Arroyo Vázquez, M. ${ }^{a}$ Luz \& Sagredo Santos, Antonia, (2008): Los Estados Unidos en sus documentos. Madrid, Universidad Nacional de Educación a Distancia, pp. 235-253):

Los mexicanos establecidos hoy en territorios pertenecientes antes a México y que quedan para lo futuro dentro de los límites señalados por el presente Tratado a los Estados Unidos, podrán permanecer en donde ahora habitan; o trasladarse en cualquier tiempo a la República mexicana, conservando en los indicados territorios los bienes que poseen, o enajenándolos y pasando su valor a donde les convenga, sin que por esto pueda exigírseles ningún género de contribución, gravamen o impuesto.

Los que prefieran permanecer en los indicados territorios podrán conservar el título y derechos de ciudadanos de los Estados Unidos.

${ }^{15}$ Heinz Kloss (1997) [1977]: The American Bilingual Tradition. United States, Center for Applied Linguistics \& Delta Systems Co. Inc., p. 20.

${ }^{16}$ George P. Hammond \& Thomas C. Donnelly (1941) [1936]: The Story of New Mexico. Albuquerque, University of New Mexico Press, p. 128.

${ }^{17}$ En 1966 pasó a llamarse College of Santa Fe.

18 Kathleen P. Chamberlain (2002): «Billy the Kid, Susan McSween, Thomas Catorn, and the Modernization of New Mexico, 1865-1912,» en Richard W. Etulain et al. (2002): New Mexican Lives. Albuquerque: University of New Mexico Press, p. 196. 
Con la llegada del ferrocarril en la década de 1880, numerosos misioneros baptistas, metodistas y presbiterianos empezaron a ocuparse de la educación y de la salud de los nuevomexicanos ${ }^{19}$. Todos los esfuerzos parecían pocos pues en 1870, el ochenta por ciento de la población nuevomexicana era analfabeta ${ }^{20}$. A lo largo de todos los años del Gobierno Territorial fueron muchas las excusas de Washington para no aceptar a Nuevo México como estado: algunas fueron de carácter formal, relacionadas con los plazos para presentar las solicitudes; otras fueron más abiertamente racistas contra una población mayoritariamente no caucásica. Otra de las razones que argumentaba el Gobierno de la Unión es que los nuevomexicanos no estaban preparados para leer y entender las leyes del estado. El Senador Arthus Beveridge, tras una visita de inspección del territorio, escribió un informe en el que contaba que en una conversación que había tenido con el superintendente del distrito escolar de Socorro, éste le había confesado que no se había enterado de la muerte de Cristóbal Colón ${ }^{21}$.

Las críticas a la ignorancia de los nuevomexicanos no eran nuevas. Josiah Gregg, comerciante, explorador y naturalista del siglo XIX ya había apuntado algo similar en 1844. Sin embargo, destacaba la corrección con la que los nuevomexicanos hablaban español:

Sin embargo, a pesar de ese terrible estado de ignorancia sobre todos esos asuntos que un hombre debería saber, es realmente sorprendente la corrección con la que la gente común habla su lengua madre, el español. El uso de palabras fuera de su sentido clásico puede darse, pero el mal uso de las reglas gramaticales (que es tan frecuente entre los analfabetos que hablan inglés) es extremadamente raro. ${ }^{22}$

En 1891, se pasó el primer Decreto de Educación Pública (Public Education Act), el primer sistema de enseñanza serio en Nuevo México, gracias al gobernador Bradford Prince. Amado Chaves fue el primer superintendente del estado y, junto a él, se creó un comité directivo del estado. Cuando se promulgó este decreto, había menos de 500 maestros en todo el estado para un total de 22.600 estudiantes aunque los que iban a la escuela con regularidad no llegaban a 15.000. En las ciudades los cursos duraban entre 8 y 9 meses pero en la zona rural podían durar tres o cuatro meses ${ }^{23}$. Gran parte del currí-

${ }^{19}$ Una de las redes más importantes de hospitales y centros de salud en Nuevo México sigue llamándose Presbyterian y Menaul School, la escuela más antigua de Albuquerque todavía en funcionamiento, fue fundada en 1896 por el reverendo presbiteriano James A. Menaul (http://www.menaulschool.com/about/the_history_ of_menaul/). Acceso el 26 de abril de 2014.

${ }^{20}$ George P. Hammond \& Thomas C. Donnelly (1941) [1936]: The Story of New Mexico. Albuquerque, University of New Mexico Press, p. 188.

${ }^{21}$ John B. Mondragón \& Ernest S. Stapleton (2005): Public Education in New México. Albuquerque, University of New Mexico, p. 18.

${ }^{22}$ Citado en Thomas E. Chávez (2002) [1992]: An Illustrated History of New Mexico. Albuquerque, University of New Mexico Press, p. 98.

Texto original:

Yet, notwithstanding this dreadful state of ignorance on all those subjects which it behooves man to be acquainted with, it is truly astonishing to notice the correctness with which common people speak their mother tongue, the Spanish. The application of words out of their classical sense may occasionally occur, but a violation of the simple grammatical rules (which is so common among the illiterate who use the English language), is extremely rare.

${ }^{23}$ George P. Hammond \& Thomas C. Donnelly (1941) [1936]: The Story of New Mexico. Albuquerque, University of New Mexico Press, p. 141. 
culo se daba en inglés y en español. La nueva ley no prohibía la enseñanza en español. De hecho, en los distritos escolares donde sólo se había hablado español, los profesores debían además tener conocimientos de ambas lenguas ${ }^{24}$. El artículo 7 del Decreto de Educación Pública indica que una de las obligaciones del superintendente es recomendar los mejores libros de texto en inglés o en inglés y en español.

En 1907, Hiram Hadley, primer anglo superintendente de Instrucción Pública en Nuevo México indica que es mejor que todos los libros de texto sean ya solamente en inglés:

Hasta 1907, no se había generalizado un esfuerzo para asegurarnos de la enseñanza del inglés en todas las escuelas públicas de nuestro territorio, pero parece que ha llegado el momento adecuado para introducir todos los libros de texto en inglés y para introducir una ley en la que todas las escuelas utilicen los mismos libros. Apenas existe oposición a esta política de enseñar sólo en inglés en la escuela primaria donde los niños van a clase por temporadas muy cortas y solamente durante unos años. Nuestras escuelas públicas no pueden ofrecer una ventaja mayor a los niños de padres hispanos que una completa formación en inglés. ${ }^{25}$

En 1912, Nuevo México se convierte oficialmente en estado de la Unión. Algunas de las provisiones de la nueva Constitución ${ }^{26}$ definen la condición de estado bilingüe de Nuevo México:

El Artículo XX, Apartado 12, por ejemplo, dice que «Todas las leyes aprobadas por la legislatura deben estar publicadas en inglés y en español durante los veinte primeros años.» ${ }^{27}$

En el Artículo XIX, Apartado 1, se indica que cualquier enmienda de la Constitución debe ser publicada en inglés y en español. ${ }^{28}$ En este mismo artículo, la Constitución se preocupa no sólo del español sino también de las lenguas indígenas y de otras lenguas que se pudieran hablar en el estado, demostrando su carácter plurilingüe y multicultural:

El Secretario de Estado también deberá hacer los suficientes esfuerzos para proveer el contenido y el propósito de las enmiendas constitucionales aprobadas por la Legislatura en las lenguas indígenas y a todos los grupos de lenguas minoritarias para informar a los electores de las enmiendas. ${ }^{29}$

${ }^{24}$ Compilation of the School Laws of the Territory of NM (1903): Article 13, Santa Fe, El Boletín Popular Printing Company, p. 5.

${ }_{25}$ Citado en Erlinda Gonzales-Berry \& David R. Maciel (2000): The Contested Homeland, Albuquerque, University of New Mexico Press, p. 172.

Texto original:

Until 1907, no general effort was made to secure the teaching of English in all of the public schools of our territory, but it seems that the time was ripe for the introduction of all English text-books and the enforcement of the uniform text-book law. Almost no opposition exists to the policy of having only English taught in the elementary schools where children attend for short terms and only for a few years. No greater advantage can be given by our public schools to the children of Spanish-American parents than a thorough training in English.

${ }_{26}$ Constitution of the State of New Mexico, 21 de enero de 1911.

27 Ibídem.

Texto original:

For the first twenty years after this constitution goes into effect all laws passed by the legislature shall be published in both the English and Spanish languages and thereafter such publication shall be made as the legislature may provide.

${ }^{28}$ Ibídem.
${ }^{29}$ Ibídem.
Texto original: 
El Artículo XXI, sección 4 (Escuelas Públicas) suponía la obligación de la enseñanza en inglés en la escuela pública:

Se debe proveer el establecimiento y el mantenimiento de un sistema de escuelas públicas que debe estar abierto a todos los niños del estado y debe ser libre de todo control sectario y dichas escuelas deben ser llevadas siempre en inglés. ${ }^{30}$

Pero el Artículo XII, Apartado 8 expresa que debe haber una adecuada preparación del profesorado en inglés y en español para una correcta enseñanza a los niños y para facilitar la enseñanza del inglés. ${ }^{31}$

El Artículo XII, Apartado 10 dice que los estudiantes de descendencia española

«... no deben ser asignados a escuelas separadas, bajo ninguna circunstancia, sino que deben gozar siempre de una perfecta igualdad con otros niños en todas las escuelas públicas e Instituciones Educativas del Estado...» ${ }^{32}$

En el Artículo XII, Apartado 11, el Spanish-American Normal School en El Rito, al norte de Española, que había sido fundado en 1909 con la intención de formar a maestros de habla hispana para trabajar con el amplio número de estudiantes hispanohablantes del territorio $^{33}$, se convierte en una institución del estado y pasa a llamarse Northern New Mexico State School (Escuela Estatal del Norte de Nuevo México). ${ }^{34}$

En 1912, la ley estableció, también, un mínimo de 5 meses por curso. Hasta entonces la ley sólo contemplaba escuelas primarias pero a partir de 1912, se potenció el que cada condado tuviera una escuela secundaria ${ }^{35}$.

En lo que a la educación superior se refiere, en 1888 se creó la institución privada de Las Cruces College. Al año siguiente, se pasó un decreto para fundar la Universidad Estatal en Albuquerque.

Entre 1915 y 1919, se hicieron las primeras propuestas para conseguir una educación bilingüe. En 1915, aprovechando el auge de la lengua española tras la apertura del Canal de Panamá, se pasó una medida propuesta por A. A. Sena, del condado de San Miguel,

The secretary of state shall also make reasonable efforts to provide notice of the content and purpose of legislatively approved constitutional amendments in indigenous languages and to minority language groups to inform electors about the amendments.

${ }^{30}$ Constitution of the State of New Mexico, 21 de enero de 1911.

Texto original:

Provision shall be made for the establishment and maintenance of a system of public schools which shall be open to all the children of the state and free from sectarian control, and said schools shall always be conducted in English.

31 Constitution of the State of New Mexico, 21 de enero de 1911.

32 Ibídem.

Texto original:

«... they shall never be classed in separate schools, but shall forever enjoy perfect equality with other children in all public schools and educational institutions of the state...»

${ }^{33}$ George P. Hammond \& Thomas C. Donnelly (1941) [1936]: The Story of New Mexico. Albuquerque, University of New Mexico Press, pp. 141-142.

${ }^{34}$ Constitution of the State of New Mexico, 21 de enero de 1911.

35 George P. Hammond \& Thomas C. Donnelly (1941) [1936]: The Story of New Mexico. Albuquerque, University of New Mexico Press, p. 141. 
que fue introducida en los estatutos de Nuevo México en la que se introducía el español aunque se dejaba claro que los libros de texto debían seguir siendo en ingles ${ }^{36}$.

El gobernador Larrazolo propuso que el español fuera enseñado en las escuelas como puente para llegar al conocimiento del inglés ${ }^{37}$. El español no se hizo obligatorio pero todo aquel que lo deseara, tenía derecho a que se le enseñara. El español se enseñaba, además, en todas las escuelas públicas de $5^{\circ}$ a $8^{\circ}$ curso.

A pesar de todas estas iniciativas, Nuevo México no se libró de la decidida política de Washington para crear la nación uniforme de la que hablaba Theodore Roosevelt, la nación de Washington y Lincoln, de Muhlenberg y Sheridan; una nación con una sola lengua, la lengua inglesa.

En 1919, la State Board of Education (la mesa directiva de educación del Estado) lo dejaba claro en su comunicado en el que el español pasaba a ser una lengua extranjera en el territorio de Nuevo México:

Se debe enseñar en inglés. La mesa directiva de Educación del Estado pasó la siguiente resolución en su sesión más reciente: La ley de nuestro estado obliga a que todas sus ramas de educación enseñadas en nuestras escuelas deben llevarse a cabo en inglés y en este sentido interpreta correctamente el espíritu y el propósito de nuestras instituciones. Esto no excluye la enseñanza de las lenguas extranjeras, lo cual es de hecho recomendable, pero es contrario al establecimiento y el mantenimiento de escuelas primarias donde todas las asignaturas se enseñan en una lengua extranjera y donde la lengua de nuestro país, que es el inglés, no se enseña lo más mínimo. ${ }^{38}$

A pesar de lo establecido en el Artículo XII, Apartado 10, de la Constitución, comenzó también la segregación de los estudiantes de origen hispano:

Durante las dos primeras décadas del siglo XX, las escuelas del Suroeste sufrían una segregación. Desde California hasta Texas, incluyendo Nuevo México, muchos mexicanoamericanos eran escolarizados de forma separada. Estudios que fueron realizados durante esos años documentan políticas abiertas de segregación en escuelas de todo el suroeste donde la lengua y la cultura de los mexicano-americanos eran consideradas inferiores. ${ }^{39}$

${ }^{36}$ Erlinda Gonzales-Berry \& David R. Maciel (2000): The Contested Homeland, Albuquerque, University of New Mexico Press, p. 177.

${ }^{37}$ Ibídem.

38 Ibídem, p. 183.

Texto original:

Must Teach in English. The State Board of Education passed the following resolution at its recent session: The law of our state is mandatory in its provisions to the effect that all branches of learning taught in our schools be so taught in the English language, and in this regard it properly interprets the spirit and purpose of our institutions. This does not exclude the teaching of foreign languages, which indeed is quite commendable, but it certainly is opposed to the establishment and maintenance of primary schools where all branches of study are taught in a foreign language, and where the language of our country, which is the English language, is only incidentally taught.

39 Joseph Edward Sweeney (1998): ¿Qué pasó?: A History of Mexican American Students in Public School Education in Central New Mexico since the Implementation of Title VII, PhD dissertation, Saint John's University in New York, pp. 17-18.

Texto original:

For the first two decades of the twentieth century, schools throughout the Southwest were segregated. From California to Texas, including New Mexico, many Mexican American children were placed in separate schools. Studies which were done during these years have documented open policies of segregation in schools throughout the Southwest where, the Mexican-American language and culture were perceived as inferior. 
A lo largo del siglo XX, una serie de decisiones en los tribunales de diversos estados y una serie de leyes han logrado paliar la discriminación que suponía la política federal de uniformidad. La primera batalla legal se ganó en Nebraska en 1920. Este estado, junto con Iowa y Ohio, habían pasado unas leyes que prohibían la enseñanza de un idioma que no fuera el inglés hasta que un niño no hubiera pasado el octavo curso. Meyer, un maestro de Nebraska, fue llevado a los tribunales por enseñar alemán a un muchacho de diez años. Fue declarado culpable. Apeló al Tribunal Supremo de Nebraska y siguió siendo considerado culpable. Fue entonces al Tribunal Supremo de los Estados Unidos. El Tribunal Supremo le dio la razón: «La protección de la Constitución se extiende a todos, a los que hablan otros idiomas igual que a los que nacieron con el inglés en la boca.» ${ }^{40}$

El Tribunal Supremo concluía:

Es de todos sabido que el dominio total de una lengua extranjera rara vez se consigue si no te enseñan desde una edad temprana y la experiencia nos dice que ni es dañina para la salud, ni para la moral ni para el entendimiento de un niño normal. ${ }^{41}$

En 1970, la ley de los Derechos Civiles decía:

Donde el hecho de no saber hablar y no poder entender la lengua inglesa impida que los niños pertenecientes a minorías no puedan participar con efectividad en el programa educativo ofrecido por un distrito escolar, el distrito debe tomar medidas compensatorias para rectificar esa deficiencia lingüística y, de esta manera, abrir sus programas de instrucción a estos estudiantes. ${ }^{42}$

En 1974, gracias al contencioso legal Lau v. Nichols, los abogados de Lau demostraron que los muchachos chinos estaban siendo discriminados en las escuelas públicas de San Francisco porque todas las clases se daban en inglés. La sentencia decidió que se estaban privando de derechos a los muchachos chino-parlantes según lo establecido en el Artículo VI de la ley de los Derechos Civiles. Esta decisión en los tribunales desencadenó la aprobación del Equal Educational Oportunity Act (ley de Oportunidades Iguales en la Educación) en 1982:

Ningún estado negará la igual oportunidad educativa a un individuo debido a su raza, color, sexo u origen nacional (...) y todas las agencias educativas tomarán las medidas adecuadas para superar las barreras lingüísticas que puedan impedir la igual participación de los

${ }^{40}$ Bill Piatt (1990): ¿Only English? Law \& Language Policy in the United States. Albuquerque, University of New Mexico Press, p.41.

Texto original:

«...the protection of the Constitution extends to all, to those who speak other languages as well as to those born with English on the tongue. »

${ }^{41}$ Ibídem.

Texto original:

«It is well known that proficiency in a foreign language seldom comes to one not instructed at an early age and experience shows that this is not injurious to the health, morals, or understanding of the ordinary child.»

${ }_{42}$ Bill Piatt (1990): ¿Only English? Law \& Language Policy in the United States. Albuquerque, University of New Mexico Press, p.43.

Texto original:

Where inability to speak and understand the English language excludes national origin minority group children from effective participation in the educational program offered by a school district, the district must take affirmative steps to rectify the language deficiency in order to open its instructional program to these students. 
estudiantes en sus programas de instrucción. ${ }^{43}$

En Nuevo México, la mayor segregación tuvo lugar en el este del Estado (esa zona es conocida como Little Texas, la pequeña Texas), aunque podríamos encontrar ejemplos de segregación por todo el estado. Un buen ejemplo fue el condado de McKinley, en el noroeste del estado, donde jamás se contrató a un maestro hispano a pesar de que más del $50 \%$ de la población era hispana ${ }^{44}$.

Hubo que esperar hasta 1973 para que la resolución judicial del caso «Serna contra Portales» confirmara el primer ejemplo de discriminación contra los mexicano-americanos, al demostrar que el distrito escolar de Portales, Nuevo México, no había logrado proveer igualdad de oportunidades educativas para los niños hispanohablantes ${ }^{45}$.

La mayoría de estas decisiones legales se consiguieron a partir de los años setenta del siglo XX. Se necesitaron dos generaciones de hispanos para reivindicar una imagen más favorable del mexicano-americano. ${ }^{46}$ La primera, más silenciosa, la denominada «Generación Mexicano-americana» sería aquella de los hispanos del suroeste entre los años 1930 y 1960; la segunda sería el famoso «Movimiento Chicano» de los años sesenta y setenta, mucho más atrevido y reivindicativo que su antecesor.

La Generación Mexicano-americana fue muy efectiva a la hora de conseguir cambios económicos, políticos, sociales, legales y educativos a través de un asociacionismo sólido en una época en la que las condiciones sociales y económicas no eran fáciles: durante la Gran Depresión, siguiendo una política claramente racista, alrededor de medio millón de mexicanos fueron deportados a México $^{47}$, siendo un buen número de ellos ciudadanos legales de los Estados Unidos. Aún con todo, la Generación Mexicanoamericana supo agruparse y nos encontramos con las primeras asociaciones mexicanoamericanas que consiguen ciertos logros sociopolíticos a través del asociacionismo.

En 1929, tuvo lugar un congreso de activistas políticos latinos en Corpus Christi, Texas, en el que se gestó la League of United Latin American Citizens, LULAC (Liga de ciudadanos latinoamericanos unidos). En su constitución, la LULAC abogaba por el uso del bilingüismo y el orgullo étnico entre los latinos. Para el comienzo de la Segunda

${ }^{43}$ Citado y explicado en Bill Piatt (1990): ¿Only English? Law \& Language Policy in the United States. Albuquerque, University of New Mexico Press, pp.43-45.

Texto original:

No state shall deny equal educational opportunity to an individual on account of his or her race, color, sex, or national origin, by (...) the failure by an educational agency to take appropriate action to overcome language barriers that impede equal participation by its students in instructional programs.

${ }^{44}$ Erlinda Gonzales-Berry \& David R. Maciel (2000): The Contested Homeland, Albuquerque, University of New Mexico Press, p. 90.

45 Joseph Edward Sweeney (1998): ¿Qué pasó?: A History of Mexican American Students in Public School Education in Central New Mexico since the Implementation of Title VII, PhD dissertation, Saint John's University in New York, p. 18.

${ }^{46}$ Gran parte de la información sobre los movimientos mexicano americanos y los movimientos chicanos han sido obtenidas en la tesis de Sweeney: Joseph Edward Sweeney (1998): ¿Qué pasó?: A History of Mexican American Students in Public School Education in Central New Mexico since the Implementation of Title VII, PhD dissertation, Saint John's University in New York, pp. 19-31.

${ }^{47}$ Antonio J. Goñi Mues (revisada en febrero 2008) [Enero 2006]: El español del suroeste de Estados Unidos: Aspectos sociolingüísticos, Memoria de Máster en Formación de Profesores de Español como Lengua Extranjera. Universitat de Barcelona, Departament de Didàctica de la Llengua i la Literatura, p. 26. 
Guerra Mundial, la LULAC tenía más de ochenta sedes por todo el suroeste, incluyendo unas cuantas en Nuevo México.

La misión de la LULAC es mejorar las oportunidades de todos los hispanos en los Estados Unidos que buscan libertad y una forma de vida honrada ${ }^{48}$. Busca mejorar la calidad de vida de los latinos en Estados Unidos y, una de las formas más efectivas de conseguirlo es a través de la mejora de las oportunidades educativas de los hispanos. En 1930, la LULAC utilizó los tribunales para denunciar la segregación que se llevaba a cabo en el distrito escolar de Del Río, en Texas. Su lucha no ha cesado desde entonces.

El Congreso del Pueblo de Habla Española fue otro movimiento nacido en los años treinta, en este caso, con más conciencia de clase trabajadora y con unos objetivos de mejora del estatus socioeconómico de los mexicano-americanos. Desde LULAC, el Congreso se veía con recelo porque tenía una ideología de extrema izquierda asociada al Partido Comunista de los Estados Unidos. Planearon su primer congreso en la Universidad de Nuevo México en 1938 pero tuvo que ser trasladado a Los Ángeles debido al carácter conservador de la universidad en esos años. Allí decidieron apoyar la creación de departamentos académicos en las universidades para el estudio de la cultura y la historia latinas. La organización fue desmantelada en 1942 debido a su ideología de izquierdas pero contribuyó de forma importante a los avances del movimiento político mexicano-americano.

La Segunda Guerra Mundial supuso un parón para estos movimientos. Sin embargo, después del conflicto bélico internacional, los veteranos de guerra de Nuevo México pasaron a formar parte del American GI Forum ${ }^{49}$, creado para cambiar la imagen de los latinos en el suroeste. Se ha dedicado, también, con mucho éxito, a llevar a los tribunales casos de discriminación escolar en el estado de Texas.

También poco después de la Guerra, en 1949, se formó en Nuevo México el partido político ANMA (Asociación Nacional Mexicana Americana), que comenzó defendiendo a los mineros nuevomexicanos de la brutalidad policial pero que continuó apoyando a las clases desfavorecidas y trabajó también en el apoyo de veteranos de guerra, en programas de educación y en cuestiones de salud pública ${ }^{50}$.

En 1896, la decisión del Tribunal Supremo en el caso Plessy v. Ferguson ${ }^{51}$ había institucionalizado y había dado legitimidad a todo tipo de prácticas segregacionistas. En 1954, la decisión en el caso Brown v. The Board of Education, Topeka, Kansas ${ }^{52}$,

${ }^{48}$ Esta organización todavía existe y puede visitarse su página web en inglés, http://lulac.org/ o en español, http://www.lulac.org/Espanol/. La misión aparece escrita en la portada de su página web. Acceso a ambas direcciones el 26 de abril de 2014.

${ }^{49}$ http://www.agifusa.org/ es la página de esta asociación creada en 1848 por el doctor Héctor Pérez García, médico militar veterano. American GI Forum presume de ser la organización de veteranos hispanos más numerosa con presencia en 40 estados y en Puerto Rico. En el 2013, su página web está solamente en inglés. Acceso el 26 de abril de 2014.

${ }^{50}$ Erlinda Gonzales-Berry \& David R. Maciel (2000): The Contested Homeland, Albuquerque, University of New Mexico Press, pp. 88-89.

51 «Plessy v. Ferguson, 163 U. S. 537 (1896)» Puede encontrarse en:

http://caselaw.lp.findlaw.com/scripts/getcase.pl?court=US\&vol=163\&invol=537. Acceso el 26 de abril de 2014.

${ }^{52}$ Podemos encontrar la decisión judicial del caso Brown v. The Board of Education (1954), Topeka, Kansas, en M. . Luz Arroyo Vázquez \& Antonia Sagredo Santos, (2008): Los Estados Unidos en sus documentos, Madrid, Universidad Nacional de Educación a Distancia, pp. 691-698. 
consiguió anular la política segregacionista y se ordenó el establecimiento de igualdad de oportunidades para todas las minorías del país.

En los años sesenta, los movimientos en defensa de los derechos civiles de la población afroamericana pronto tuvieron sus réplicas dentro de la población hispana. Las protestas multitudinarias contra la guerra de Vietnam unieron al pueblo americano. Todas las razas estaban implicadas en la guerra y las protestas contra la guerra fueron el detonante de numerosos movimientos que defendían una mayor igualdad de oportunidades para todas las razas, todos los sexos y todas las clases sociales.

El Movimiento Chicano, mucho más agresivo y contundente que los movimientos anteriores, englobó a grupos como La Alianza, La Raza Unida, The Crusade for Justice, The United Farm Workers ${ }^{53}$ y múltiples movimientos estudiantiles chicanos. Todos combatieron para que los hispanos tuvieran un mayor reconocimiento social, la cultura y la historia de los latinos tuviera mayor presencia en el currículum y los muchachos de la raza tuvieran las mismas oportunidades que todos los demás ciudadanos del país. Algunas veces, la lucha fue armada, como en el caso de La Alianza que, liderada por el texano Reies López Tejerina, tomó los juzgados en Tierra Amarilla, Nuevo México, por la fuerza en 1967, denunciando que el gobierno estadounidense y algunos particulares tenían que devolver la tierra que habían robado a los nuevomexicanos. Los miembros de la Alianza intercambiaron disparos con las fuerzas del orden y, al final, los juzgados fueron recuperados con la ayuda del ejército. Más específicamente involucrada con la educación, apareció MALDEF ${ }^{54}$ (The Mexican American Legal Defense and Education Fund - Fondo para la educación y la defensa legal de los mexicano-americanos) todavía activa en la actualidad. MALDEF se opone explícitamente a las políticas del «English Only» o «American English» y apoya la ley de No Child Left Behind a pesar de que admite que a veces no está bien implementada.

El MEChA ${ }^{55}$ (Movimiento Estudiantil Chicano de Aztlán) se estableció como una de las coaliciones de asociaciones estudiantiles chicanas a finales de los años sesenta. La nueva generación prefería el término chicano a la denominación de mexicano-americanos. El MEChA consiguió que numerosas universidades del suroeste incluyeran estudios hispanos en los currículos.

Es en este contexto de movilizaciones sociales cuando se aprobó la ley nacional de la Educación bilingüe (Bilingual Education Act) en 1968. La ley fue primero escrita por el senador por Texas, Ralph Yarborough, como presidente del Subcomité Especial sobre la educación bilingüe. El senador por Nuevo México, Joseph Montoya, apoyó el proyecto desde el comienzo, y encontró a un experto en la materia y defensor de la educación bilingüe, Henry Pascual, dentro del mismo estado. Cuando el senador Yarborough perdió su escaño en 1968, fue Montoya el que tomó el liderazgo en la defensa e implementación de la ley de Educación Bilingüe, mejor conocida como «Título VII» (Title VII) que fue aprobada en ese mismo año. El presidente de los Estados Unidos,

${ }^{53} \mathrm{http} / / / \mathrm{www}$.ufw.org/, en inglés, español y tailandés. Acceso el 26 de abril de 2014. Fue la asociación del movimiento obrero que fundó César Chávez. Dolores Huerta, cofundadora de la asociación, nació en Dawson, Nuevo México en 1930.

${ }^{54} \mathrm{http}: / /$ www.maldef.org/, su página web está solamente en inglés. Acceso el 26 de abril de 2014.

${ }^{55}$ http://www.nationalmecha.org/, su página web está solamente en inglés. Acceso el 26 de abril de 2014. 
Lyndon B. Johnson, firmó el Título VII y lo convirtió en ley. El Título VII se justificaba a sí mismo debido a la necesidad de ayudar a víctimas de la pobreza que tenían problemas para hablar en inglés.

La enmienda a la ley nacional de 1974 añadía también un componente cultural e histórico al documento final del Título VII. La Ley Nacional de Educación Bilingüe fue reautorizada por quinta y última vez en $1994^{56}$ y fue transformada en el 2002 en la Ley de Adquisición de la Lengua Inglesa dentro del famoso paquete legal No Child Left Behind $^{57}$, la ley de Educación del gobierno de George W. Bush.

La ley de Educación Bilingüe admite que «la presencia de americanos con lenguas minoritarias está relacionada sólo en parte con la política federal de inmigración», lo cual implica la aceptación de que hay lenguas minoritarias propias del territorio estadounidense. El propósito de la ley era el siguiente:

(8) es el propósito de este título ayudar a asegurar que estudiantes con un dominio del inglés limitado aprendan correctamente el inglés y alcancen altos niveles académicos en las asignaturas de contenidos.

(9) los programas con calidad de educación bilingüe ayudan a los niños y muchachos a aprender inglés y conseguir altos resultados académicos incluyendo el dominio de más de una lengua;

(10) conforme el mundo es cada vez más interdependiente y la comunicación internacional se convierte en algo cotidiano en el gobierno, los negocios, el comercio y la vida familiar, las habilidades políglotas constituyen un importante recurso nacional que merece ser protegido y desarrollado. ${ }^{58}$

Nuevo México fue el primer estado en pasar una ley estatal de educación bilingüe en 1969 (House Bill 75 y Senate Bill 270) ${ }^{59}$. El objetivo fundamental era el mantenimiento de la lengua y de la cultura de los niños del Estado. Roberto Mondragón llevó la iniciativa en la Cámara Baja y Jerry Apodaca en el Senado. En 1973 se enmendó la ley original de 1969 y se pasó la ley de Educación bilingüe vigente en el 2010 (Senate Bill 421). 700,000 dólares fueron destinados a la implementación de esta ley para su primer año, en el que se debía aplicar para los cursos de primaria de primero a sexto. En 1987, la ley se extendió para que, progresivamente fuera aplicándose a los cursos superiores,

${ }^{56}$ Versión de la Ley de Educación Bilingüe la última vez que fue reautorizada. Puede encontrarse en: http://www.nabe.org/Resources/Documents/Advocacy\%20page/TitleVII1994.pdf. Acceso el 26 de abril de 2014.

57 «Que Ningún Niño Se Quede Atrás» es la traducción que ofrece el Ministerio de Educación de los Estados Unidos, US Education Department: Véase en:

http://www2.ed.gov/espanol/bienvenidos/es/nclb.html. Acceso el 26 de abril de 2014.

${ }^{58}$ Ley de Educación Bilingüe (1994) [1968]: Parte de la SEC. 7102. FINDINGS, POLICY, AND PUR$P O S E$ de la ley.

Texto original:

'(8) it is the purpose of this title to help ensure that limited English proficient students master English and develop high levels of academic attainment in content areas;

(9) quality bilingual education programs enable children and youth to learn English and meet high academic standards including proficiency in more than one language;

(10) as the world becomes increasingly interdependent and as international communication becomes a daily occurrence in government, business, commerce, and family life, multilingual skills constitute an important national resource which deserves protection and development;

${ }^{59}$ Nos estamos refiriendo a las dos cámaras del Gobierno de Nuevo México, la cámara de representantes o «House» y el Senado y a los números que se otorgaron a la ley en ambas cámaras) 
para que en el curso académico 1990-91, todos los niveles de educación pública fueran cubiertos por la ley de Educación bilingüe del Estado.

Las leyes de Educación Bilingüe hicieron grandes progresos. Algunos de los logros de todo este movimiento a favor de las lenguas minoritarias desde la aprobación del Título VII fueron la aprobación de la licencia educativa de la lengua navajo en 1986, por la que se establecían los requisitos necesarios para poder enseñar esa lengua; la declaración del estado de Nuevo México como territorio partidario del English Plus en $1989^{60}$; la creación en 1990 del componente bilingüe en las licencias de los maestros que quisieran enseñar alguna de las lenguas de los indios Pueblo; la firma del Memorándum de entendimiento entre el gobierno de Nuevo México y el de España en 1990; la firma del Memorándum de entendimiento entre el gobierno de Nuevo México y el de México en 199461; la creación de La Prueba en 1994, con la que se examinaba a los maestros que querían añadir a su licencia el componente bilingüe en español (y por lo tanto, aumentar su sueldo); el pilotaje de programas duales en 1996; En 2001, se fortalecieron los programas de educación bilingüe en las lenguas nativo americanas y se regularon las licencias alternativas que podían conseguir los maestros de estas lenguas americanas; la Asamblea Legislativa de Nuevo México destinó fondos para tres nuevas maneras de «Prueba de Español para la certificación bilingüe» en 2005; y la declaración de Nuevo México, por parte del gobernador, como un Estado multicultural en el 2006.

La ley de Educación Bilingüe supuso también un buen revulsivo económico para los Ministerios de Educación de los estados con más estudiantes cuya lengua madre no era el inglés, siendo Nuevo México un estado altamente beneficiado por el programa al tener un buen número de hispanohablantes y un buen número de hablantes de lenguas nativo americanas. El gasto federal en educación bilingüe pasó de 7.500.000 de dólares en 1968 a 150 millones de dólares en $1979^{62}$. De 1995 al año 2000, el gobierno federal repartió unos 215 millones de dólares anuales para fortalecer estos programas.

Mientras tanto, la educación bilingüe es uno de los temas candentes de la actualidad norteamericana. Esto se entiende perfectamente cuando se descubre que alrededor del $47 \%$ del presupuesto del estado de Nuevo México va a la educación pública ${ }^{63}$ (lo máximo que ha puesto el gobierno federal en el gasto de educación del estado es el 10\%). Sin embargo, ni los defensores de la educación bilingüe ni los que están en su contra tienen muy claro las leyes que están apoyando o denigrando y son partidarios, más bien, del espíritu de esas ideologías en vez de conocer a fondo la ley.

De hecho, la Ley de Educación Bilingüe, la federal, es un marco legal muy similar al de una declaración de derechos civiles. Esta ley incitó a la mayoría de los estados, a la promulgación de leyes de educación bilingüe estatales. Cada estado intentó aprovechar al máximo los fondos que venían acompañados de la ley federal a través de las leyes es-

\footnotetext{
${ }^{60}$ Puede encontrarse en la página web del Ministerio de Educación de Nuevo México:

http://www.ped.state.nm.us/BilingualMulticultural/d109/New\%20Mexico\%20--\%20English\%20Plus\%20 Resolution.pdf. Acceso el 26 de abril de 2014.

${ }^{61}$ Los dos memorándum facilitan los intercambios educativos de maestros y profesores de secundaria a la vez que promueven iniciativas educativas conjuntas entre Nuevo México y estos dos países de lengua hispana.

${ }_{62}$ David Frum (2000): How We Got Here: The '70s. New York, Basic Books, pp. 270-271.

${ }_{63}$ John B. Mondragón \& Ernest S. Stapleton (2005): Public Education in New México. Albuquerque, University of New Mexico, p. 32.
} 
tatales pero éstas diferían de estado en estado dependiendo de las políticas más o menos conservadoras de cada uno de ellos. En muchos casos, las políticas anduvieron errantes dependiendo de los cambios de gobierno en cada estado y de la fluidez de los fondos que estos iban recibiendo.

Como hemos visto, la educación bilingüe en Nuevo México se remonta al siglo XVI y llega a nuestros días. Muchos han sido los logros que han llevado a conseguir que el estado siga siendo plurilingüe y multicultural. Muchos son los retos, económicos, sociales y políticos que seguirán marcando el destino de la educación bilingüe en Nuevo México y en el conjunto de los Estados Unidos. 
